\title{
The Effect of Animation Realism on Face Ownership and Engagement
}

\author{
Elena Kokkinara* $\quad$ Rachel McDonnell ${ }^{\dagger}$ \\ Graphics, Vision, and Visualisation Group \\ Trinity College Dublin
}
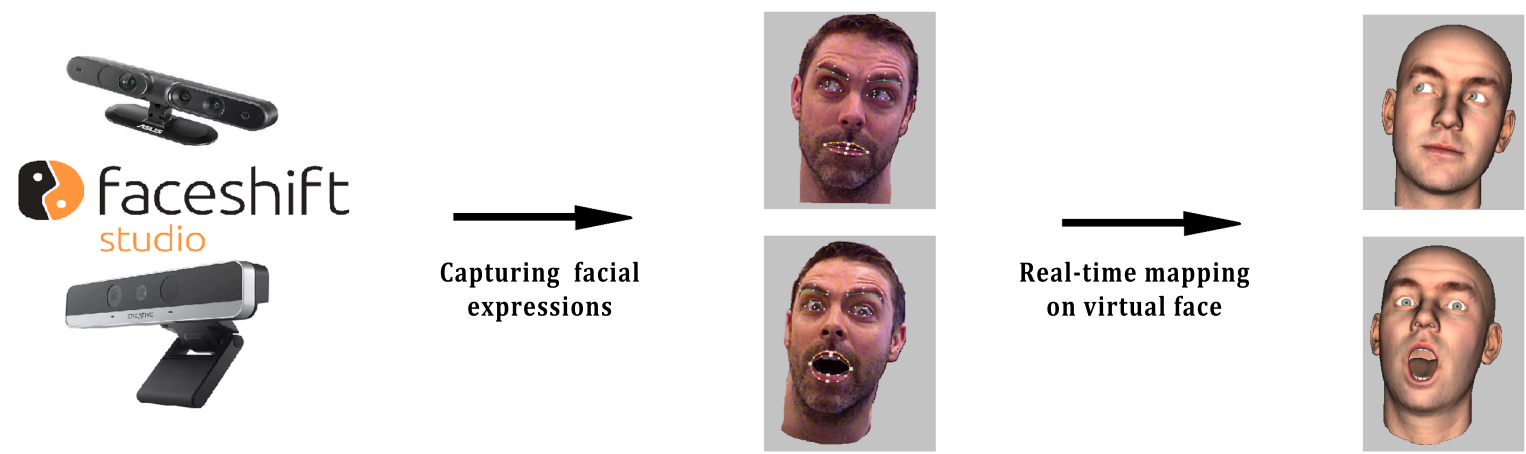

Figure 1: Tracking data is mapped on virtual face's expressions in real-time, using FaceShift studio. We measure participants' feeling of ownership and engagement over the virtual face.

\section{Introduction}

Recent advances in facial tracking technologies have allowed us to create realistic animations of virtual faces that would function even in real-time. A number of systems have recently been developed for gaming and VR platforms, mainly aiming to track actors' expressions and using them for off-line editing.

Animation realism of virtual characters' faces has been considered highly important for conveying emotions and intent [Hyde et al. 2013]. However, to our knowledge, no perceptual experiments have assessed the way that participants engage with their own animated virtual face and what are the influencing factors, when they see a real-time mirrored representation of their facial expressions mapped on the virtual face.

Studies in immersive virtual environments have shown that it is possible to feel an illusory ownership over a virtual body, when the body is seen from a fist person perspective and when participants receive synchronous tapping on the virtual body and their hidden real body [Slater et al. 2009]. Similarly, when participants see their collocated virtual body animating in synchrony with their tracked real body, they can feel a sense of ownership and control over their virtual representation [Kokkinara and Slater 2014]. Here, we consider the possibility to perceive ownership and control over a mirrored virtual face with synchronous animated expressions to the tracked real face.

\section{Experiment}

We conducted a preliminary study, where 15 male participants interacted with an abstract-looking AI agent speaking in a robot voice on a screen-based virtual environment. The AI agent influenced the interaction in a way that would provoke head movements, speaking and facial expression from the participant. Head movements and expressions were tracked in real-time and were mapped on a realistic-looking virtual face model using the FaceShift tool (Figure 1).

\footnotetext{
*e-mail:ekokkina@scss.tcd.ie

†email:ramcdonn@scss.tcd.ie
}

A set of standardized questionnaires were used to assess the levels of perceived level of appeal, ownership and control over the virtual face, as well as the levels of engagement with the AI agent during the experience. Questions were rated on a Likert scale from 1 (totally disagree) to 7 (totally agree).

\section{Results}

Participants reported high levels of ownership towards the virtual face [median (interquartile range - IQR) $=5(0.25)]$, agency [me$\operatorname{dian}(\mathrm{IQR})=7(1)]$, ownership of the movements [median(IQR) $=6(1)]$, character appeal $[$ median $(\mathrm{IQR})=5(0.25)]$ and enjoyment interacting with the AI agent $[$ median $(\mathrm{IQR})=5(2)]$.

In a follow up control condition participants will receive asynchronous feedback, using pre-recorded facial animations. We expect that participants will engage less and feel lower levels of ownership with the asynchronously animated face, compared to the synchronous condition tested here.

\section{Conclusion}

This study can provide valuable insights regarding engagement with self-avatars and facial animation. Observing believable representations of our own facial expressions on self-avatars can potentially have a big effect on an actor's performance and engagement with their role, while it can even drive the design of future games incorporating real-time facial animation.

\section{References}

Hyde, J., Carter, E. J., Kiesler, S., And Hodgins, J. K. 2013. Perceptual effects of damped and exaggerated facial motion in animated characters. In Automatic Face and Gesture Recognition (FG), 2013 10th IEEE International Conference and Workshops on, IEEE, 1-6.

Kokminara, E., AND Slater, M. 2014. Measuring the effects through time of the influence of visuomotor and visuotactile synchronous stimulation on a virtual body ownership illusion. Perception 43, 1, 43-58.

Slater, M., Perez-Marcos, D., Ehrsson, H. H., And Sanchez-Vives, M. V. 2009. Inducing illusory ownership of a virtual body. Frontiers in neuroscience 3 , $2,214$. 